MMCA-97 Conference, pp. 122-129

R. Čiegis (Ed)

(C) 1997 Vilniaus Gedimino technikos universitetas

\title{
POLYNOMIAL SPLINE COLLOCATION METHOD FOR NONLINEAR TWO-DIMENSIONAL WEAKLY SINGULAR INTEGRAL EQUATIONS
}

\author{
ARVET PEDAS \\ Institute of Applied Mathematics, \\ University of Tartu, \\ J.Liivi Str. 2-206, Tartu EE2400, Estonia
}

\section{INTRODUCTION}

The solution of a second kind Fredholm integral equation with weakly singular kernel is typically nonsmooth near the boundary of the domain of integration (its derivatives are unbounded, see, for example, [1-3, 5, 7-8, 10-14]). If one wants to obtain a high order convergence of a numerical method for these equations one has to take into account, in some way, the singular behavior of the exact solution. The purpose of the present paper is to discuss how it can be done using polynomial splines on special graded grids in the numerical solution of a sufficiently wide class of nonlinear two-dimensional weakly singular integral equations.

\section{INTEGRAL EQUATION}

We consider the nonlinear equation

$$
u(x)-\int_{G} K(x, y, u(y)) d y=f(x), \quad x \in G,
$$

where

$$
G \equiv\left\{x=\left(x_{1}, x_{2}\right): 0<x_{1}<d_{1}, 0<x_{2}<d_{2}\right\} \subset \mathbb{R}^{2} .
$$

The kernel $K(x, y, u)$ is assumed to be $m$ times $(m \geq 1)$ continuously differentiable with respect to $x, y$ and $u$ for $x \in G, y \in G, x \neq y, u \in \mathbb{R}$, whereby there exists a real number $\nu \in \mathbb{R}, \nu<2$, such that, for any nonnegative integer $k \in \mathbb{Z}_{+}$and multi-indexes $\alpha=\left(\alpha_{1}, \alpha_{2}\right) \in \mathbb{Z}_{+}^{2}$ and $\beta=\left(\beta_{1}, \beta_{2}\right) \in \mathbb{Z}_{+}^{2}$ 
with $k+|\alpha|+|\beta| \leq m$, the following inequalities hold:

$$
\begin{gathered}
\left|D_{x}^{\alpha} D_{x+y}^{\beta}\left(\frac{\partial}{\partial u}\right)^{k} K(x, y, u)\right| \leq b_{1}(|u|)\left\{\begin{array}{ll}
1, & \nu+|\alpha|<0 \\
1+|\log | x-y||, & \nu+|\alpha|=0 \\
|x-y|-\nu-|\alpha|, & \nu+|\alpha|>0
\end{array} ;\right. \\
\left|D_{x}^{\alpha} D_{x+y}^{\beta}\left(\frac{\partial}{\partial u}\right)^{k} K\left(x, y, u_{1}\right)-D_{x}^{\alpha} D_{x+y}^{\beta}\left(\frac{\partial}{\partial u}\right)^{k} K\left(x, y, u_{2}\right)\right| \leq \\
\leq b_{2}\left(\max \left\{\left|u_{1}\right|,\left|u_{2}\right|\right\}\right)\left|u_{1}-u_{2}\right| \begin{cases}1, & \nu+|\alpha|<0 \\
1+|\log | x-y||, & \nu+|\alpha|=0 \\
|x-y|^{-\nu-|\alpha|}, & \nu+|\alpha|>0\end{cases}
\end{gathered}
$$

Here $|\alpha|=\alpha_{1}+\alpha_{2}$ for $\alpha \in \mathbb{Z}_{+}^{2},|x|=\left(x_{1}^{2}+x_{2}^{2}\right)^{1 / 2}$ for $x \in \mathbb{R}^{2}$,

$$
D_{x}^{\alpha}=\left(\frac{\partial}{\partial x_{1}}\right)^{\alpha_{1}}\left(\frac{\partial}{\partial x_{2}}\right)^{\alpha_{1}}, \quad D_{x+y}^{\beta}=\left(\frac{\partial}{\partial x_{1}}+\frac{\partial}{\partial y_{1}}\right)^{\beta_{1}}\left(\frac{\partial}{\partial x_{2}}+\frac{\partial}{\partial y_{2}}\right)^{\beta_{2}}
$$

and the functions $b_{1}:[0, \infty) \rightarrow[0, \infty)$ and $b_{2}:[0, \infty) \rightarrow[0, \infty)$ are assumed to be monotonously increasing.

Note that the assumptions (2) and (3) hold, for example, for the kernels

$$
K(x, y, u)=K_{1}(x, y, u)|x-y|^{-\gamma}
$$

and

$$
K(x, y, u)=K_{1}(x, y, u) \log |x-y|
$$

where $0<\gamma<2$ and $K_{1}(x, y, u)$ is a $m+1$ times continuously differentiable function with respect to $x, y, u$ for $x, y \in \bar{G}=\left\{\left(x_{1}, x_{2}\right): 0 \leq x_{1} \leq d_{1}, 0 \leq\right.$ $\left.x_{2} \leq d_{2}\right\}, u \in \mathbb{R}$ (see (2) and (3) with $\nu=\gamma$ and $\nu=0$, respectively). In the case $\nu<0$ the kernel $K(x, y, u)$ is bounded but its derivates may be singular.

For the right hand term of equation (1), we assume that $f \in C^{m, \nu}(G)$ (with the same $m$ and $\nu$ as in (2) and (3)). The space $C^{m, \nu}(G)$ is defined as the collection of all $m$ times continuously differentiable functions $u: G \rightarrow \mathbb{R}$ such that

$$
\|u\|_{m, \nu} \equiv \sum_{|\alpha| \leq m} \sup _{x \in G}\left(\tau_{|\alpha|-(2-\nu)}(x)\left|D_{x}^{\alpha} u(x)\right|\right)<\infty
$$

Here

$$
\tau_{\lambda}(x)=\left\{\begin{array}{ll}
1, & \lambda<0 \\
{[1+|\log \varrho(x)|]^{-1},} & \lambda=0 \\
\varrho(x)^{-\lambda}, & \lambda>0
\end{array}\right\}, \quad x \in G, \quad \lambda \in \mathbb{R}
$$


and $\varrho(x)=\inf _{y \in \partial G}|x-y|$ is the distance from $x \in G$ to $\partial G$, the boundary of G.

\section{SMOOTHNESS OF THE SOLUTION}

The following results state the regularity properties of a solution $u \in L^{\infty}(G)$ of equation (1) and give a basis for a discussion of the optimal (global and local) order of convergence of piecewise polynomial collocation methods for equation (1) (see Sections 3 and 4).

TheOrem $1[14,7] \quad$ Let $f \in C^{m, \nu}(G)$, and let the kernel $K(x, y, u)$ satisfy conditions (2) and (3). If integral equation (1) has a solution $u \in L^{\infty}(G)$, then $u \in C^{m, \nu}(G)$, i.e., the growth of its derivatives $D_{x}^{\alpha} u(x)$ near the boundary $\partial G$ can be estimated as follows:

$$
\begin{aligned}
& \left|D_{x}^{\alpha} u(x)\right| \leq \mathrm{const}\left\{\begin{array}{ll}
1, & |\alpha|<2-\nu \\
1+|\log \varrho(x)|, & |\alpha|=2-\nu \\
\varrho(x)^{2-\nu-|\alpha|}, & |\alpha|>2-\nu
\end{array}\right\} \\
& (x \in G,|\alpha| \leq m) .
\end{aligned}
$$

Denoting, for $x \in G$,

$$
\varrho_{k}(x)=\min \left\{x_{k}, d_{k}-x_{k}\right\}, \quad k=1,2,
$$

we have $\varrho(x)=\min \left\{\varrho_{1}(x), \varrho_{2}(x)\right\}$. Introduce the space $C_{\square}^{m, \nu}(G)$ consisting of functions $u \in C^{m, \nu}(G)$ such that

$$
\begin{aligned}
& \left|\frac{\partial^{l} u(x)}{\partial x_{k}^{l}}\right| \leq \operatorname{const}\left\{\begin{array}{ll}
1, & l<2-\nu \\
1+\left|\log \varrho_{k}(x)\right|, & l=2-\nu \\
\varrho_{k}(x)^{2-\nu-l}, & l>2-\nu
\end{array}\right\}, \\
& x \in G ; l=1, \ldots, m ; k=1,2 .
\end{aligned}
$$

Theorem $2[14,8]$. Let $f \in C_{\square}^{m, \nu}(G)$, and let the kernel $K(x, y, u)$ satisfy conditions (2) and (3). If integral equation (1) has a solution $u \in L^{\infty}(G)$ then $u \in C_{\square}^{m, \nu}(G)$.

Note that the estimates (4) and (5) for a solution $u$ of equation (1) on the conditions of Theorem 2 are sharp in many cases. This complicates an effective solution of the integral equation (1). In Section 3 the piecewise polynomial collocation method is presented which is adapted to the possible singularities of the solution and is of the same accuracy as if there were no singularities in the solution at all. Moreover, in Section 4, using special collocation points, error estimates at the collocation points are given, showing a more rapid convergence as the global uniform convergence in $G$ available by piecewise polynomials. 
Notice also that $C^{m}(\bar{G}) \subset C^{m, \nu}(G) \subset C_{\square}^{m, \nu}(G)$, where $C^{m}(\bar{G})$ is the space of $m$ times continuously differentiable functions $u: \bar{G} \rightarrow \mathbb{R}$. On the other hand, a function $u \in C_{\square}^{m, \nu}(G)$ always can be extended up to a continuous function on $\bar{G}$.

\section{COLLOCATION METHOD}

To introduce the partition of $\bar{G}$ into cells we choose the vector $N=\left(N_{1}, N_{2}\right)$ of natural numbers and introduce in the intervals $\left[0, d_{k}\right], k=1,2$, the following $2 N_{k}+1$ grid points:

$$
\begin{array}{ll}
x_{k, N}^{j_{k}}=\frac{d_{k}}{2}\left(\frac{j_{k}}{N_{k}}\right)^{r}, & j_{k}=0,1, \ldots, N_{k} ; \\
x_{k, N}^{N_{k}+j_{k}}=d_{k}-x_{k, N}^{N_{k}-j_{k}}, & j_{k}=1, \ldots, N_{k} .
\end{array}
$$

Here $r \in \mathbb{R}, r \geq 1$, characterizes the degree of the non-uniformity of the grid (6). If $r=1$ then the grid points (6) are uniformly located.

Denote

$$
J_{N} \equiv\left\{j=\left(j_{1}, j_{2}\right): j_{k}=1, \ldots, 2 N_{k} ; k=1,2\right\} .
$$

Using points (6) we introduce the partition of $\bar{G}$ into closed cells $G_{N}^{j}$ :

$$
G_{N}^{j} \equiv\left\{x=\left(x_{1}, x_{2}\right): x_{k, N}^{j_{k}-1} \leq x_{k} \leq x_{k, N}^{j_{k}}\right\} \subset \bar{G}, \quad j \in J_{N} .
$$

We determine the collocation points in the following way. We choose $m$ points $\eta_{1}, \ldots, \eta_{m}$ in the interval $[-1,1]$ :

$$
-1 \leq \eta_{1}<\ldots<\eta_{m} \leq 1
$$

By affine transformations we transfer them into the interval $\left[x_{k, N}^{j_{k}-1}, x_{k, N}^{j_{k}}\right]\left(j_{k}=\right.$ $\left.1, \ldots, 2 N_{k} ; k=1,2\right)$ :

$$
\xi_{k, N}^{j_{k}, q_{k}}=x_{k, N}^{j_{k}-1}+\frac{\eta_{q_{k}}+1}{2}\left(x_{k, N}^{j_{k}}-x_{k, N}^{j_{k}-1}\right), \quad q_{k}=1, \ldots, m .
$$

Note that $\xi_{k, N}^{j_{k}-1, m}=\xi_{k, N}^{j_{k}, 1}=x_{k, N}^{j_{k}}$ if $\eta_{1}=-1, \eta_{m}=1\left(j_{k}=1, \ldots, 2 N_{k}-1\right)$. We assign the collocation points

$$
\begin{gathered}
\xi_{N}^{j, q}=\left(\xi_{1, N}^{j_{1}, q_{1}}, \xi_{2, N}^{j_{2}, q_{2}}\right), \quad q \in Q, \\
Q \equiv\left\{q=\left(q_{1}, q_{2}\right): q_{k}=1, \ldots, m ; k=1,2\right\},
\end{gathered}
$$

to the cells $G_{N}^{j}, j \in J_{N}$. 
For a function $u: \bar{G} \rightarrow \mathbb{R}$ we construct a piecewise polynomial interpolation function $P_{N} u: \bar{G} \rightarrow \mathbb{R}$ as follows:

1) on every cell $G_{N}^{j}, j \in J_{N}, P_{N} u$ is a polynomial of the degree not exceeding $m-1$ with respect to both of arguments $x_{1}$ and $x_{2}$;

2) $P_{N} u$ interpolates $u$ at points $\xi_{N}^{j, q}, q \in Q$. Thus, $P_{N} u$ is uniquely defined in every cell $G_{N}^{j}$ separately and may have jumps on the hyperplanes $x_{k}=x_{k, N}^{j_{k}}$, $j_{k}=1, \ldots, 2 N_{k}-1$. We may treat $P_{N} u$ as a multivalued function on these hyperplanes. In the case $\eta_{1}=-1, \eta_{m}=1, P_{N} u$ is a continuous function on $\bar{G}$.

Let us denote by $E_{N}$ the range of the interpolation projector $P_{N}$. This is the finite dimensional space of piecewise polynomial functions $u_{N}$ on $\bar{G}$ which on any cell $G_{N}^{j}, j \in J_{N}$, are polynomials of degree not exceeding $m-1$ with respect to any of arguments $x_{1}$ and $x_{2}$.

The approximate solution $u_{N} \in E_{N}$ of the integral equation (1) we determine by the collocation method from the following conditions:

$$
\left[u_{N}(x)-\int_{G} K\left(x, y, u_{N}(y)\right) d y-f(x)\right]_{x=\xi_{N}^{i, p}}=0, \quad p \in Q, i \in J_{N} .
$$

We can define $u_{N} \in E_{N}$ by the formula

$$
u_{N}(x)=\sum_{q \in Q} c_{N}^{j, q} \varphi_{N}^{j, q}(x), \quad x \in G_{N}^{j}, \quad j \in J_{N}
$$

where

$$
\varphi_{N}^{j, q}(x)=\varphi_{1, N}^{j_{1}, q_{1}}\left(x_{1}\right) \varphi_{2, N}^{j_{2}, q_{2}}\left(x_{2}\right)
$$

and $\varphi_{k, N}^{j_{k}, q_{k}}\left(x_{k}\right), k=1,2$, are the polynomials of one variable of degree $m-1$ such that

$$
\varphi_{k, N}^{j_{k}, q_{k}}\left(\xi_{k, N}^{j_{k}, p_{k}}\right)=\left\{\begin{array}{ll}
1 & \text { if } p_{k}=q_{k} \\
0 & \text { if } p_{k} \neq q_{k}
\end{array}\right\}, \quad p_{k}=1, \ldots, m
$$

Now the collocation conditions (10) will take the following form of a nonlinear system which determines the coefficients $c_{N}^{j, q}=u_{N}\left(\xi_{N}^{j, q}\right)$ :

$$
c_{N}^{i, p}=\sum_{j \in J_{N}} \int_{G_{N}^{j}} K\left(\xi_{N}^{i, p}, y, \sum_{q \in Q} c_{N}^{j, q} \varphi_{N}^{j, q}(y)\right) d y+f\left(\xi_{N}^{i, p}\right), \quad p \in Q, \quad i \in J_{N} .
$$

If $\eta_{1}>-1$ or $\eta_{m}<1$ then all collocation points $\xi_{N}^{j, q}, q \in Q, j \in J_{N}$, are different and there are $(2 m)^{2} N_{1} N_{2}$ collocation points. Thus, in this case the system (10') has $(2 m)^{2} N_{1} N_{2}$ equations and the same number unknowns. If $\eta_{1}=-1, \eta_{m}=1$ then part of the collocation points coincide. The number of 
different collocation points is $\left(2 N_{1}(m-1)+1\right)\left(2 N_{2}(m-1)+1\right)=\operatorname{dim} E_{N}$ and the system (10') has the same number of equations and unknowns.

THEOREM 3 [14]. Let the following conditions be fulfilled:

1) graded grid (6) and collocation points (9) are used;

2) kernel $K(x, y, u)$ satisfies (2) and (3)

3) $f \in C_{\square}^{m, \nu}(G)$;

4) integral equation (1) has a solution $u_{0} \in L^{\infty}(G)$ whereby the linearized equation

$$
v(x)=\int_{G} K_{0}(x, y) v(y) d y, \quad K_{0}(x, y)=\left.\frac{\partial K(x, y, u)}{\partial u}\right|_{u=u_{0}(y)},
$$

has in $L^{\infty}(G)$ only the trivial solution $v=0$.

Then there exist $N_{k}^{0}>0(k=1,2)$ and $\delta_{0}>0$ such that, for $N_{k} \geq N_{k}^{0}$ $(k=1,2)$, the collocation conditions (10) define a unique approximation $u_{N} \in E_{N}$ to $u_{0}$ satisfying $\left\|u_{N}-u_{0}\right\|_{L^{\infty}(G)} \leq \delta_{0}$. The following error estimates hold:

$$
\begin{aligned}
& \sup _{x \in G}\left|u_{N}(x)-u_{0}\right| \leq \text { const } h^{m} \text { for }\left\{\begin{array}{ll}
r>\frac{m}{2-\nu} & \text { if } 2-\nu<m \\
r>1 & \text { if } 2-\nu=m \\
r \geq 1 & \text { if } 2-\nu>m
\end{array}\right\} ; \\
& \varepsilon_{N} \leq \text { const } h^{m} \text { for }\left\{\begin{array}{lll}
r>\frac{m}{2(2-\nu)}, & r \geq 1 & \text { if } 2-\nu \leq 1 \\
r>\frac{m}{2-\nu+1} & \text { if } 1<2-\nu \leq m-1 \\
r \geq 1 & \text { if } 2-\nu>m-1
\end{array}\right\},
\end{aligned}
$$

where

$$
h=\max \left\{\frac{d_{1}}{N_{1}}, \frac{d_{2}}{N_{2}}\right\}
$$

and

$$
\varepsilon_{N}=\max _{q \in Q, j \in J_{N}}\left|u_{N}\left(\xi_{N}^{j, q}\right)-u_{0}\left(\xi_{N}^{j, q}\right)\right|
$$

is the maximal error of $u_{N}$ at collocation points (9).

Note that an estimate $\sup _{x \in G}\left|u_{N}(x)-u(x)\right|=\mathcal{O}\left(h^{m}\right)$ is of optimal order even for a function $u \in C^{\infty}(\bar{G})$. Theorem 3 shows that, for the collocation method (10), the accuracy $\mathcal{O}\left(h^{m}\right)$ can be achieved using sufficiently great values of the scaling parameter $r$ of the grid (6). There are possibilities to reduce $r$ restricting ourselves to uniform estimates at the collocation points only (see (15)). Moreover, using special collocation points, the accuracy $\varepsilon_{N}=o\left(h^{m}\right)$ can be achieved (see next Section). Thus, the superconvergence phenomenon at collocation points takes place $[4,14,9,6]$. 


\section{SUPERCONVERGENCE AT THE COLLOCATION POINTS}

Now we assume that $\eta_{1}, \ldots, n_{m}$ (see (7)) are the nodes of a quadrature formula

$$
\int_{-1}^{1} \varphi(\xi) d \xi \approx \sum_{k=1}^{m} w_{k} \varphi\left(\eta_{k}\right), \quad-1 \leq \eta_{1}<\ldots<\eta_{m} \leq 1
$$

which is exact for all polynomials of degree $m+\mu, \mu \in \mathbb{Z}, 0 \leq \mu \leq m-$ 1. Actually, the weights $w_{1}, \ldots, w_{m}$ of the quadrature formula (18) will not be used in our discussion. The case $\mu=m-1$ corresponds to the Gauss quadrature formula and is of the greatest interest in the following theorem.

THEOREM 4 [6]. Let the following conditions be fulfilled:

1) kernel $K(x, y, u)$ and $\partial K(x, y, u) / \partial u$ are $m+\mu+1$ times $(m, \mu \in \mathbb{Z}$, $m \geq 1,0 \leq \mu \leq m-1)$ continuously differentiable with respect to $x, y$ and $u$ for $x \in G, y \in G, x \neq y, u \in \mathbb{R}$, and satisfy (2) and (3) for $|\alpha|+|\beta|+k \leq m+\mu+1$ with a $\nu \in \mathbb{R}, \nu<2$;

2) $f \in C_{\square}^{m+\mu+1, \nu}(G)$;

3) equation (1) has a solution $u_{0} \in L^{\infty}(G)$ and the linearized equation (13) has in $L^{\infty}(G)$ only the trivial solution $v=0$;

4) graded grid (6) is used with the scaling parameter $r=r(m, \nu, \mu) \geq 1$ satisfying the corresponding restrictions in (14) strenghend to the strict inequality $r>m /(2-n u)$ if $2-\nu<\mu+1$, and the additional conditions

$$
\left\{\begin{array}{ll}
r \geq \frac{m+2-\nu}{3-\nu} & \text { if } 2-\nu<\mu+1 \\
r>\frac{m+\mu+1}{3-\nu} & \text { if } 2-\nu \geq \mu+1
\end{array}\right\}
$$

5) collocation points (9) are generated by the nodes (7) of a gvadrature formula (18) which is exact for all polynomials of degree $m+\mu, 0 \leq \mu \leq m-1$.

Then

$$
\begin{gathered}
\varepsilon_{N} \leq \operatorname{const} h^{m}\left[\begin{array}{ll}
h^{\mu+1}, & 2-\nu>\mu+1 \\
h^{\mu+1}(1+|\log h|), & 2-\nu=\mu+1 \\
h^{2-\nu}, & 2-\nu<\mu+1
\end{array}\right\} \\
\left.+\left\{\begin{array}{ll}
h^{2}, & \nu<0 \\
h^{2}(1+|\log h|), & \nu=0 \\
h^{2-\nu}, & \nu>0
\end{array}\right\}\right]
\end{gathered}
$$

where $h$ and $\varepsilon_{N}$ are defined in (16) and (17), respectively.

Acknowledgment. This work was partly supported by the Estonian Science Foundation (Research Grant No. 2999). 


\section{REFERENCES}

[1] I.G. Graham. Singularity expansions for the solutions of second kind Fredholm integral equations with weakly singular convolution kernels. J. Integral Equations, 4, 1982, P. 1-30.

[2] W. Hackbusch. Integralgleichungen. Theorie und Numerik, Teubner, Stuttgart, 1989.

[3] H. Kaneko, R. Noren, Y. Xu. Regularity of the solution of Hammerstein equations with weakly singular kernel. Integral Equations Operator Theory, 13, 1990, P. 660670.

[4] H. Kaneko, R. Noren, Y. Xu. Numerical solutions for weakly singular Hammerstein equations and their superconvergence. J. Integral Equations Appl., 4, 1992, P. 391 406.

[5] U. Kangro. The smoothness of the solution to a two-dimensional integral equation with logarithmic kernel. Z. Anal. Anw., 12, 1993, P. 305-318.

[6] A. Pedas. On the superconvergence of a spline-collocation method for nonlinear two-dimensional weakly singular integral equations. Submitted for publication "J. Differential Equations". (In Russian).

[7] A. Pedas, G. Vainikko. The smoothness of solutions to nonlinear weakly singular integral equations. Z. Anal. Anw., 13(3), 1994, P. 463-476.

[8] A. Pedas, G. Vainikko. Tangential derivatives of solutions to nonlinear multidimensional weakly singular integral equations. In: Beitrage zur Angewandten Analysis und Informatik. Helmut Brakhage zu Ehren (Hrsg. Eberhard Schock), Scbaker Verlag, Aachen, 1994, P. 271-287.

[9] A. Pedas, G. Vainikko. Superconvergence of a piecewise polynomial collocation method for nonlinear weakly singular integral equations. Submitted for publication "J. Integral Equations Appl."

[10] J. Pitkäranta. Estimates for derivates of solutions to weakly singular Fredholm integral equations. SIAM J. Math. Anal. 11, 1980, P. 952-968.

[11] G.R. Richter. On weakly singular integral equations with displacement kernels. $J$. Math. Anal. Appl., 55, 1976, P. 32-42

[12] C. Scheider. Regularity of the solution to a class of weakly singular Fredholm integral equations of the second kind. Integral Equations Operator Theory, 2, 1979 , P. 62-68.

[13] P. Uba. The smoothness of the solution of weakly singular equations with a discontinuous coefficient. Proc. Eston. Acad. Sci. Phys., Math. 37(2), 1988, P. 192-203. (in Russian).

[14] G. Vainikko. Multidimensional Weakly Singular Integral Equations. Lecture Notes in Mathematics, 1549, Springer Verlag, Berlin-Heidelberg, 1993. 